Bundesgesundheitsbl 2018 - 61:1030-1031 https://doi.org/10.1007/s00103-018-2775-2 Online publiziert: 25. Juni 2018

(c) Springer-Verlag GmbH Deutschland, ein Teil von Springer Nature 2018

CrossMark

Robert Koch-Institut

Robert Koch-Institut, Berlin, Deutschland

\title{
Erratum zu: Liste der vom Robert Koch-Institut geprüften und anerkannten Desinfektionsmittel und-verfahren
}

\section{Stand 31. Oktober 2017 (17. Ausgabe)}

\section{Erratum zu:}

Bundesgesundheitsblatt 2017

https://doi.org/10.1007/s00103-017-

2634-6

Dieses Erratum beinhaltet Berichtigungen zu Verfahren bzw. Produkten, die in der oben angegebenen Ausgabe nicht korrekt dargestellt wurden.
1.S. 1274

1. Thermische Verfahren

\subsection{Verbrennen}

Wirkungsbereich: ABCD

2. S. $1278 \bullet$ Tab. 2

\begin{tabular}{|c|c|c|c|c|c|c|c|c|c|c|c|c|c|}
\hline \multirow[t]{4}{*}{$\begin{array}{l}\text { Wirk- } \\
\text { stoff }\end{array}$} & \multirow[t]{4}{*}{ Name } & \multicolumn{2}{|c|}{$\begin{array}{l}\text { Flächendes- } \\
\text { infektion } \\
\text { (Wischdes- } \\
\text { infektion) }\end{array}$} & \multicolumn{2}{|c|}{$\begin{array}{l}\text { Wäschedes- } \\
\text { infektion } \\
\text { (Einlege- } \\
\text { verfahren) }\end{array}$} & \multicolumn{6}{|c|}{$\begin{array}{l}\text { Desinfektion von Ausscheidungen } 1 \text { Teil } \\
\text { Auswurf oder Stuhl + } 2 \text { Teile GV bzw. } 1 \text { Teil } \\
\text { Harn + } 1 \text { Teil GV }\end{array}$} & \multirow[t]{4}{*}{$\begin{array}{l}\text { Wirkungs- } \\
\text { bereich }\end{array}$} & \multirow[t]{4}{*}{$\begin{array}{l}\text { Herstel- } \\
\text { ler bzw. } \\
\text { Liefer- } \\
\text { firma }\end{array}$} \\
\hline & & \multirow{3}{*}{$\begin{array}{l}\text { GV } \\
\text { [\%] }\end{array}$} & \multirow{3}{*}{$\begin{array}{l}\text { EWZ } \\
\text { [min] }\end{array}$} & \multirow{3}{*}{$\begin{array}{l}\text { GV } \\
{[\%]}\end{array}$} & \multirow{3}{*}{$\begin{array}{l}\text { EWZ } \\
\text { [h] }\end{array}$} & \multicolumn{2}{|c|}{ Auswurf } & \multicolumn{2}{|c|}{ Stuhl } & \multicolumn{2}{|c|}{ Harn } & & \\
\hline & & & & & & GV & EWZ & GV & EWZ & GV & EWZ & & \\
\hline & & & & & & [\%] & [min] & [\%] & [min] & [\%] & [min] & & \\
\hline & $\begin{array}{l}1+1 \text { Wofasteril SC } \\
\text { super-Kombi- } \\
\text { verfahren }{ }^{2} \\
\text { Wofasteril SC super } \\
\text { und alcapur }\end{array}$ & $\begin{array}{l}2 \\
2\end{array}$ & 60 & & & & & & & & & $A B$ & $\begin{array}{l}\text { Kesla } \\
\text { Pharma }\end{array}$ \\
\hline & $\begin{array}{l}\text { Wofasteril-Kombi- } \\
\text { verfahren } \\
\text { Wofasteril und } \\
\text { alcapur }\end{array}$ & $\begin{array}{l}2 \\
6\end{array}$ & 60 & & & & & & & & & $A B$ & $\begin{array}{l}\text { Kesla } \\
\text { Pharma }\end{array}$ \\
\hline
\end{tabular}

Die Online-Version des Originalartikels ist unter https://doi.org/10.1007/s00103-017-2634-6zu finden. 
3. S. 1296

Anhang zur Liste der vom Robert Koch-Institut geprüften und anerkannten Desinfektionsmittel und-verfahren

\section{Korrektur von Produktnamen}

Änderung von

- Wofasteril E400 in Wofasteril

- alkapur $\mathrm{N}$ in alcapur $\mathrm{N}$

\section{Korrespondenzadresse}

Robert Koch-Institut

Robert Koch-Institut

Nordufer 20, 13353 Berlin, Deutschland 